\title{
Les Méthodes Moléculaires Pour La Caractérisation Des Champignons Phytopathogènes
}

\section{Hamida Benslimane, PhD}

Ecole Nationale Supérieure d'Agronomie, Département de Botanique, Laoratoire de Pytopatologie et Biologie Moléculaire, Alger, Algérie

doi: 10.19044/esj.2016.v12n24p234 URL:http://dx.doi.org/10.19044/esj.2016.v12n24p234

\begin{abstract}
Development of new disease-resistant varieties and the development of epidemic control strategies, require knowledge of the molecular structure and genetic relationships among the pathogen populations. Molecular methods are used to explore the diversity at the DNA level. In fact, they highlight molecular markers which are independent of the host selection, allowing study of biodiversity on the genome. These markers are most effective than a virulence markers, consequently they has become widely used in the plant pathology laboratories. This mini-review summarized the main molecular methods used; the paper describe for each one, the principle, the sequenced steps, then the advantages or inconveniences.
\end{abstract}

Keywords: Molecular markers, fungi, phytopathology

\section{Résumé}

Le développement de nouvelles variétés résistantes aux maladies et la mise au point de stratégies de contrôle des épidémies, nécessitent la connaissance de la structure moléculaire et des relations génétiques dans une population pathogène. Les méthodes moléculaires permettent en effet d'explorer la variabilité naturelle existante au niveau de l'ADN. Les marqueurs qu'elles génèrent, étant indépendants de la sélection de l'hôte, ils permettent une étude de la biodiversité directement au niveau du génome. Ils sont dans ce sens, plus efficaces que les marqueurs de virulence, et leur utilisation est ainsi devenue largement répondue dans les laboratoires de phytopathologie. Le présent article rapporte les méthodes moléculaires les plus communément rencontrées; il décrit succinctement pour chacune d'elles le principe, les étapes de réalisation et les avantages ou inconvénients que présente l'adoption de l'une ou l'autre des méthodes.

Mots clés : Marqueurs moléculaires, champignons, phytopathologie 


\section{Introduction}

Les méthodes moléculaires sont universellement applicables et permettent d'explorer le polymorphisme à différents niveaux (comparaison entre des souches, des espèces, des genres, etc.) (Verscheure et al., 2002). Elles permettent d'explorer l'énorme variabilité naturelle existante au niveau de l’ADN (Xu, 2010). Ceci s’est révélé très important dans la mesure où la connaissance des relations génétiques dans une population pathogène est indispensable pour le développement de nouvelles variétés résistantes et la mise au point de stratégie de contrôle des épidémies. En effet, malgré l'intérêt des marqueurs de virulence qui sont mis en évidence via les tests du pouvoir pathogène, ceux-ci ne révèlent qu'une petite portion de la diversité génétique présente dans une population pathogène. Par contre, il est admis que les marqueurs moléculaires qu'ils soient de type protéines ou ADN, sont indépendants de la sélection de l'hôte et permettent une étude de la biodiversité directement au niveau du génome. Cependant, les marqueurs moléculaires basés sur l'analyse de l'ADN sont actuellement préférés à ceux basés sur l'analyse des protéines lors de la caractérisation des différents champignons phytopathogènes.

\section{Méthodes basées sur l’analyse des protéines :}

Elles comportent l'étude des protéines totales, des allozymes et le plus souvent l'étude des isozymes. Il existe diverses études où cette dernière approche a été utilisée pour la différentiation et la caractérisation entre les isolats, les formes spéciales ou les races de plusieurs champignons phytopathogènes (Burdon et Marshall, 1981 ; Berner et al., 1983 ; Alfenas et al., 1984 ; Lin et al., 1989 ; Fofana et al., 1992 ; Weitz et al., 1993).

Les marqueurs de type isozymes ont montré leurs limites, car pour chaque système enzymatique, les conditions optimales d'extraction et d'utilisation des tampons doivent être déterminées. De plus, la variation intra-spécifique peut être trop faible pour être détectée avec ce type de méthode. Enfin, certains enzymes sont exprimés uniquement à des stades de développement particuliers ou peuvent être spécifiques à certains tissus (Michelmore et Hulbert, 1987).

\section{Méthodes basées sur l'analyse de l'ADN :}

Un large spectre de méthodes rentre sous la coupole des techniques basées sur l'étude de l'ADN. Parmi les plus utilisées, nous discuterons la RFPL (Restriction Fragment Lenght Polymorphism), la RAPD (Random Amplified Polymorphic DNA), l'AFLP ( Amplified Fragment Lenght ) et les microsatellites ou SSR (Simple Sequence Repeat). Chacune de ces méthodes présente des avantages et des inconvénients : 


\section{La RFPL (Restriction Fragment Lenght Polymorphism)}

Le polymorphisme de taille des fragments de restriction implique une digestion de l’ADN génomique par des enzymes de restriction, ceci génère plusieurs fragments. Les bandes sont alors identifiées individuellement grâce à une hybridation avec une sonde appropriée (Apuya et al., 1988). C’est un outil important dans l'identification de la diversité inter et intra-spécifique (Southern, 1979). Lors de l'étude d'un fragment d'ADN ou d'un gène particulier, la digestion enzymatique peut être précédée d'une PCR (Frisvad et al., 1998), nous parlons alors de la PCR-RFPL. Cette dernière approche est largement exploitée pour l'étude de plusieurs espèces fongiques ; lorsqu'elle est suivie d'un séquençage des fragments générés, elle est très utile pour les investigations afférentes à la variabilité entre souches, l'évolution des populations et celle des espèces (Xu, 2010). Dans cette optique, les régions ITS (Internal Transcribed Spacers) sont largement utilisées pour la comparaison entre espèces (Nasraoui et Lepoivre, 2003), ce sont probablement les régions les plus séquencées chez les champignons actuellement. De même, les IGS (Inter Genitic Spacer), sont plus recommandées pour l'étude de la diversité intra-spécifique car ces séquences possèdent la variabilité la plus importante observée au niveau de l'ADN ribosomique. Enfin, il est à noter que la PCR-RFLP peut générer d'excellents marqueurs co-dominants de grande stabilité et reproductibilité, idéal aussi bien pour les organismes haploïdes que diploïdes (Xu et al, 1999).

\section{La RAPD (Random Amplified Polymorphic)}

Elle consiste en l'amplification par PCR de fragments de l'ADN génomique en utilisant des amorces arbitraires de courte taille $(10 \mathrm{pb})$ (Williams et al., 1990). L'analyse via cette méthode révèle deux types de variation génétique; celle de la longueur du fragment qui sépare deux sites d'hybridation de l'amorce, et la variation dans la séquence du site d'hybridation (Xu, 2001). Cette technique est reconnue pour être simple, rapide et ne nécessite pas de connaissance préalable de la séquence. Son inconvénient majeur réside dans le fait qu'elle manque de reproductivité car elle est très sensible à la concentration en ADN et aux conditions d'amplification (Najimi et al., 2003).

Elle a été utilisée afin d'évaluer le niveau de la diversité génétique entre les races de plusieurs champignons phytopathogènes (Chen et al., 1993 ; Kolmer et al., 1995 ; Borchardt et al., 1998 ; Gonzalez et al., 1998 ; Hamelin et al., 1998). Elle a également été l'outil d'identification et de discrimination entre les pathotypes d'autres espèces (Kelly et al., 1994 ; Goodwin et Annis, 1991 ). De même qu'elle a été largement employée pour la discrimination entres isolats de provenances diverses (Vakalounakis et Fragkiadakis, 1999 ; Singh, 2001 ; Santos et al. 2002 ; Singh et Hughes, 
2006). Elle permet aussi la mise en évidence de certains marqueurs ; tel est le cas à titre d'exemple pour Pyrenophora teres f. teres chez qui un marqueur RAPD associé à une faible virulence a été identifié (Weiland et al., 1999). Enfin, elle est recommandée pour le développement d’empreinte génétique utilisé comme marqueur épidémiologique (Xu, 2010).

\section{L’AFLP (Amplified Fragment Lenght Polymorphic)}

Elle est fondée sur l'amplification sélective des fragments de restriction. Elle comporte trois étapes: (i) L'ADN génomique est clivé par deux enzymes de restriction, puis des adaptateurs de séquences connues et spécifiques des enzymes de restriction utilisées sont ajoutés aux extrémités des fragments. (ii) L’amplification sélective des fragments de restriction : une première amplification, dite pré-amplification est réalisée à l'aide d'amorces de séquences complémentaires à la séquence des adaptateurs et des sites de restriction, la deuxième amplification, dite sélective, utilise des amorces identiques aux premières mais prolongées à l'extrémité 3' de 1 à trois nucléotides; ces amorces sélectives permettent de réduire le nombre de fragments amplifiés à une centaine. (iii) L’analyse des fragments amplifiés par électrophorèse sur gel de polyacrylamide (Vos et al., 1995). Cette technique est puissante, stable et rapide, elle ne nécessite aucune connaissance préalable de séquences du génome étudié (Najimi et al., 2003). Toutefois, elle nécessite une très bonne qualité de l'ADN et un niveau de technicité important.

La technique AFLP s’est révélée être un outil très précieux dans les études des structures génétiques des champignons et leur diversité. Sa grande sensibilité génère plus d’information sur la variabilité que toute autre technique moléculaire (Majer et al., 1996). Son application a été rapportée à titre d'exemple, sur Septoria (Kema et al., 2002 ; Schnieder et al., 1998), Leptosphaeria (Pongam et al., 1999) , Fusarium (Abd-Elsalam et al., 2002) et Pyrenophora tritici-repentis (Leisovà et al., 2008 ; Friesen et al. 2005).

\section{Les microsatellites ou SSR (Simple Sequence Repeat)}

Les microsatellites sont constitués de séquences de di-, tri- ou tétranucléotides répétés en tandem. Ces éléments sont uniformément répartis en plusieurs exemplaires sur l'ensemble du génome d'une espèce et présente un taux de polymorphisme élevé. Ce dernier est dû à la variation du nombre d'unités de répétition constituant le microsatellite (Morgante et Olivieri, 1993). Par voie de conséquence, la technique SSR repose sur l'amplification de ces motifs et la détection de la différence entre le nombre de répétition chez les individus différents. Ceci est possible après le développement d'amorces complémentaires des régions flanquant le microsatellite (Field et al., 1996). Cette méthode présente l'avantage d'être reproductible et 
nécessite peu d'ADN, mais elle est longue et coûteuse (Verscheure et al., 2002).

Elle a été, à l’origine, utilisée pour évaluer la diversité chez les végétaux et les animaux puis utilisée actuellement pour l'étude des champignons (Guo, 2010). Cependant, les microsatellites des espèces fongiques semblent exhiber un polymorphisme moins important que celui des autres organismes (Dutech et al., 2007). Ainsi, la technique SSR a été employée dans plusieurs travaux traitant de diversité moléculaire d'espèces fongiques. A titre d'exemple, chez Phaeosphaeria nodorum, l'utilisation de cette méthode sur une collection de 693 isolats provenant de champs de blé des cinq continents a montré l'existence d'une très grande diversité chez cette espèce mais un faible niveau de diversité entre les groupes provenant de continents différents. De même qu’il a été possible de démontrer que la similitude génétique observée entre les populations était due au taux de migration qui a permis de réduire la différentiation des populations (Stukenbrock et al., 2006). L’amplification des microsatellites des ADN d'une collection d'Ascochyta rabiei a permis de classer les isolats en plusieurs groupes, mais aucune corrélation avec l’origine géographique n’a été retrouvé (Bayraktar et al., 2007).

\section{Conclusion}

Les phytopathologistes utilisent des techniques de laboratoire très variées; celles-ci sont aussi variables que la diversité des thématiques de cette discipline et dépendent des approches envisagées en recherche. Si les méthodes classiques ont été souvent efficaces durant plusieurs décennies, l'évolution des exigences de la recherche a nécessité par la suite, le recourt à d'autres techniques plus afférentes à première vue à d'autres disciplines, telles que la biochimie, la cytologie ou la physiologie. Aujourd'hui, le recourt aux techniques de biologie moléculaire est sans équivoque.

\section{References:}

Abd-Elsalam K.A., Khalili M.S., Aly A.A. and Asran-Amal A. (2002). Genetic diversity among Fusarium oxysporum f.sp. vasinfectum isolates revealed by UP-PCR and AFLP markers. Phytopathol. Mediterr., 41, 1-7. Alfenas A.C., Jeng R. and Hubbes M. (1984). Isozyme and proteins of isolates of Cryphonectria cubensis differing in virulence. Can. J. Bot., 62, 23-30.

Apuya N.R., Frazier B.L., Keim P., Roth E.J. and Lark K.G. (1988). Restriction fragment length polymorphism as a genetic markers in soybean, Glycine max (L.) Merrill. Theor. Appl. Genet., 75, 889-901.

Bayraktar H, Dolar F.S and Tör M. (2007). Determination of genetic diversity within Ascochyta rabei (Pass.)Labr., The case of Ascochyta Blight of chichpea in Turkey. J. Plant Pathol., 89 (3), 341-347. 
Bernier, Jeng L.R.S. and Hubbes M. (1983). Differentiation of aggressive and non-agressive isolates of Ceratocysitis ulmi by electrophoresis of intramycelial enzymes. Mycotaxon, 17, 466-472.

Borchardt D.S., Welz H.G. and Geiger H.H. (1998). Genetic structure of Setosphaeria turcica populations in tropical and temperate climates. Phytopathology, 88, 322-329.

Burdon J.J. and Marshall D.R., 1981. Isozyme variation between species and formae speciales of the genus Puccinia. Can. J. Bot, 59, 2628-2643.

Chen X, Line R.F. and Leung H. (1993). Relationship between virulence variation and DNA polymorphism in Puccinia striiformis. Phytopathology, 83, 1489-1497.

Dos Santos A.M.P.V., Matsumura A.T.S. and Vander Sand S.T. (2002). Intraspecific genetic diversity of Drechslera tritici-repentis as detected by random amplified polymorphic DNA analysis. Genet. Mol. Biol., 25, 243250.

Dutech C., Enjalbert J., Fournier E., Delmotte F., Barrès B., Carlier J., Tharreau D. and Giraud T. (2007). Challenges of microsatellite isolation in fungi. Fungal Genet. Biol., 44, 933-949.

Field D., Eggert L., Metzgar D., Rose R., and Wills C. (1996). Use of polymorphic short and clustered coding region microsatellites to distinguish strains of Candida albicans. FEMS Immunol. Med. Mic., 15, 73-79.

Frisvad J.C., Bridge P.D. and Arora D.K. (1998). Chemical fungal taxonomy. New York: Marcel Dekker.

Friesen T.L., Ali S., Klein K.K. and Rasmussen J.B. (2005). Population genetic analysis of a global collection of Pyrenophora tritici-repentis, causal agent of tan spot of wheat. Phytopathology, 95, 1144-1150.

Fofana A., Louanchi M., Robin P., Balesdent M.H and Despreaux D.(1992). Interspecific variation in soluble protein and isozyme patterns in Rigiodoporus lignosus, the causal agent of white root disease on Heavea brasiliensis. Indian J. Nat. Rubber Res. 5,141-150.

Gonzalez M., Rodriguez R., Zavala M.E., Jacobo J.L., Hernadez F., Acosta J., Martinez O. and Simpson J. (1998). Characterization of Mexican isolates of Colletotrichum lindemuthianum by using differential cultivars and molecular markers. Phytopathology, 88, 292-299.

Goodwin P.H. and Annis S.L. (1991). Rapid identification of genetic variation and pathotype of Leptosphaeria maculans by random amplified polymorphic DNA assay. Appl. Environ. Microbiol., 57, 2482-2486.

Guo L.D., (2010). Molecular diversity and Identification of endophytic fungi. In: Springer Heidelberg Dordrecht (eds.) Molecular identification of fungi. (pp. 277-296). London, England. 
Hamelin R.C., Dusabenyagasani M. and Et-touil K. (1998). Fine-level genetic structure of white pine blister rust populations. Phytopathology, 88, 1187-1191.

Kema G.H.J., Goodwin S.B., Hamza S., Verstappen E.C.P., Cavaletto J.R., Van derlee T.A.J., De Weerdt M., Bonants P.J.M. and Wlwijk C. (2002). A combined amplified fragment length polymorphism and randomly amplified polymorphism DNA genetic linkage map of Mycosphaerella graminicola, in Septoria tritici leaf blotch pathogen of wheat. Genetics, 161, 1497-1505.

Leisová L., Hanzalová A. and Kucera L. (2008). Genetic diversity of Pyrenophora tritici-repentis isolates as revealed by AFLP analysis. J. Plant Pathol., 90, 233-245.

Lin D., Dumas M.T. and Hubbes M. (1989). Isozyme and general protein patterns of Armillaria spp. Collected from the boreal mixed wood forest of Ontario. Can. J. Bot, 67, 1143-1147.

Majer D., Mitten R., Lewis B.G., Vos P. And Olivier R.P. (1996). The use of AFLP fingerprinting for the detection of genetic variation in fungi. Mycol. Res., 100, 1107-1111.

Michelmore R. W. and Hulbert S.H. (1987). Molecular markers for genetic analysis of phytopathogenic fungi. Annu. Rev. Phytopathol., 25, 383-404.

Morgante M. And Olivieri A.M. (1993). PCR-amplified microsatellites as markers in plant genetics. Plant J., 3, 175-182.

Najimi B., El Jaafari S., Jlibéne M. et Jacquemin J.M. (2003). Application des marqueurs moléculaires dans l'amélioration du blé tendre pour la résistance aux maladies et aux insectes. B.A.S.E., 7, 17- 35.

Nasraoui B. et Lepoivre P. 2003. Les champignons phytopthogènes. In : De Boeck Université (eds.), Phytopathologie :Bases moléculaires et biologiques des pathosystèmes et fondements des stratégies de lutte.(pp.111-142). Bruxelles, Belgique.

Pongam P., Osborn T.C. and Williams P.H. (1999). Assessment of genetic variation among Leptospharia maculans isolates using pathogenecity data and AFLP analysis. Plant Dis., 83, 149-154.

Singh P.K. (2001). Genetic studies on the resistance to tan spot of wheat and genetic similarity among isolates of Pyrenophora tritici-repentis. PhD thesis, University of Saskatchewan, Canada.

Singh P.K. and Hughes G.R. (2006). Genetic similarity among isolates of Pyrenophora tritici-repentis, causal agent of tan spot of wheat. $J$. Phytopathol., 154, 178-184.

Schnieder F., Koch G., Jung C., Verret J.A. (1998). The application of molecular markers for genetic characterization of Septoria tritici population. J. Pant Dis. Protect., 105 (5), 452-461.

Southern E.M. (1979). Gel electrophoresis of restriction fragments. Methods enzymol., 68, 152-76. 
Stukenbrock E.H., Banke S., and McDonald B.A. (2006). Global migration patterns in the fungal wheat pathogen Phaeosphaeria nodorum. Mol. Ecol. , 10, 1-10.

Vakalounakis D.J. and Fragkiadakis G.A. (1999). Genetic diversity of Fusarium oxysporum isolates from cucumber : Differentiation by pathogenicity vegetative compatibility, and RAPD fingerprinting. Phytopthology, 89, 161-168.

Verscheure M., Lognay G. et Marlier M. (2002). Revue bibliographique : les méthodes chimiques d'identification et de classification des champignons. B.A.S.E, 6, 131-142.

Vos, P., Hogers, R., Bleeker, M., Reijans, M., van de Lee, T., Hornes, M., Frijters, A., Pot, J., Peleman, J., Kuiper, M. and Zabeau, M. (1995). AFLP: a new technique for DNA fingerprinting. Nucleic Acids Res., 23, 4407-4414.

Weiland, J.J., SteVenson, B.J., Cartwright, R.D., Webster, R.K. (1999). Identication of molecular genetic markers in Pyrenophora teres $f$. teres associated with low virulence on 'Harbin' barley. Phytopathology , 89, 176181.

Weitz H.G., Köhler W. and Leonard K. (1993). Isozyme variation within and among pathogenic races of Cochliobolus carbonum on Corn in North California. Molecular Ecology. Phytopthathology, 84, 31-38.

Williams J.G.K., Kubelki A.R., Livak K.J., Rafalski J.A., Tingey S.V. (1990). DNA polymorphisms amplified by arbitrary primers are useful as genetic markers. Nucleic Acids Res., 18, 6531-6533.

$\mathrm{Xu}$ J. (2010). Fundamentals of Fungal Molecular Population Genetic Analysis. Curr. Issues Mol., 8, 75-90.

Xu, J., Mitchell, T.G., and Vilgalys, R.J. (1999). PCR restriction fragment length polymorphism (RFLP) analyses reveal both extensive clonality and local genetic differences in Candida albicans. Mol. Ecol., 8, 59-73. 